\title{
Expression of neutrophil gelatinase-associated lipocalin in low osmolar contrast-induced nephropathy in rats and the effect of $\mathrm{N}$-acetylcysteine
}

\author{
WEN-HUA LI*, LIN WANG*, HAI-YAN HE, JING CHEN and YA-REN YU \\ Department of Cardiology, Institute of Cardiovascular Diseases Research, Affiliated Hospital of Xuzhou Medical College, \\ Xuzhou, Jiangsu 221002, P.R. China
}

Received August 15, 2015; Accepted August 25, 2016

DOI: 10.3892/etm.2016.3779

\begin{abstract}
Serum creatinine (Scr), which is a conventional indicator of contrast-induced nephropathy (CIN), is unable to reflect the damage of kidney promptly. The present study aimed to investigate the value of neutrophil gelatinase-associated lipocalin (NGAL) in kidney and serum of CIN rats to observe whether NGAL can be used as a superior indicator of CIN. Furthermore, N-acetylcysteine (NAC) was used to assess its effect on CIN. A total of 120 adult male Sprague Dawley rats were randomly divided into four groups ( $n=30 /$ group): CIN rats (CIN), normal rats treated with NAC (NAC), CIN rats treated with $\mathrm{NAC}(\mathrm{NAC}+\mathrm{CIN})$ and the control group (CON). Serum Scr and NGAL values were measured at 2, 12, 24,48 and $72 \mathrm{~h}$ following the procedure. Immunohistochemistry and western blot analysis were used to detect NGAL within the kidney tissue. Hematoxylin and eosin staining were used to access the renal injury score. Oxidative stress within the kidney was analyzed via malondialdehyde (MDA) and superoxide dismutase (SOD). The level of NGAL in the serum and tissue of the CIN group increased significantly $2 \mathrm{~h}$ after the procedure $(\mathrm{P}<0.05)$. However, the Scr value did not exhibit a significantly change until $48 \mathrm{~h}$ later. Based on the renal injury scores, NAC reduced the kidney damage caused by the contrast. NAC treatment was associated with a decrease in SOD levels and an increase in MDA. These findings suggested that NGAL was a superior indicator of CIN than Scr, as NGAL was able to detect kidney damage much earlier. Furthermore,
\end{abstract}

Correspondence to: Professor Wen-Hua Li, Department of Cardiology, Institute of Cardiovascular Diseases Research, Affiliated Hospital of Xuzhou Medical College, 99 Huaihai West Road, Xuzhou, Jiangsu 221002, P.R. China

E-mail: xzwenhua0202@163.com

*Contributed equally

Key words: contrast-induced nephropathy, N-acetylcysteine, neutrophil gelatinase-associated lipocalin
NAC treatment inhibited oxidative stress, thus protecting against CIN.

\section{Introduction}

With the development of invasive cardiology, contrast-induced nephropathy (CIN) has become an important issue to tackle in clinical practice. It has become the third leading cause of hospital-acquired acute renal failure, accounting for $10-25 \%$ of all acquired acute renal damage (1). The traditional biomarker for diagnosis of CIN is Scr, which is not sensitive enough to detect early kidney damage, and various factors affect its accuracy (2), such as age, gender, muscle mass, diet, medication and hydration status. Neutrophil gelatinase-associated lipocalin (NGAL), known as NGAL, lipocalin 2, siderocalin, uterocalin, proteinase -3 and $24 \mathrm{p} 3$, is a mammalian small 25-kDa peptide that belongs to the lipocalin superfamily, which consists of $\sim 20$ small lipoproteins. NGAL was initially discovered as an antibacterial factor of natural immunity and an acute-phase protein (3). NGAL levels are low in normal functioning kidneys; however, when ischemic injury or nephrotoxicant occurs, NGAL accumulates at high levels in the renocortical tubule and urine (4). NGAL is readily excreted to serum and urine (5); therefore, it may be a novel marker for the early detection of CIN.

$\mathrm{N}$-acetylcysteine (NAC) is an antioxidant agent with myocardial and renal protective potential (6-8). NAC's primary use in clinical medicine has been as a mucolytic agent to treat respiratory diseases. More recently, it has been suggested that NAC has a vasodilating effect (9). Since CIN may be associated with renal vasoconstriction and oxidative stress (10-12), we hypothesize that NGAL may be a novel biomarker that can detect CIN earlier and more accurately, and that the use of NAC significantly reduces the incidence of CIN.

\section{Materials and methods}

Animals. Adult male Sprague-Dawley rats $(n=120)$, weighing $200 \pm 20$ g, were obtained from Xuzhou Medical College Laboratory Animal Center (Xuzhou, China). Rats were housed in standard stainless steel hanging cages with controlled temperature $\left(22-25^{\circ} \mathrm{C}\right)$, humidity $(35-50 \%)$, and photocycle 
(12-h light/dark) conditions. Experiments were performed in accordance with the Guide for the Care and Use of Laboratory Animals published in P.R. China and with the approval of Xuzhou Medical Ethics Committee.

Experimental design and drugs. Rats were randomly divided into four equal groups: Control (CON; n=30), CIN-treated $(\mathrm{CIN} ; \mathrm{n}=30)$, normal rats treated with NAC (NAC; $\mathrm{n}=30)$, and $\mathrm{CIN}$ rats treated with NAC (NAC+CIN; $=30)$. A total of $10 \mathrm{mg} / \mathrm{kg}$ indomethacin (INDO), $10 \mathrm{mg} / \mathrm{kg} \mathrm{Nv}$-nitro-L-arginine methyl ester (both Sigma-Aldrich; Merck Millipore, Darmstadt, Germany) and $2.9 \mathrm{~g} \mathrm{I} / \mathrm{kg}$ iohexol (Jiangsu Yangtze River Pharmaceutical Group Co., Ltd., Taizhou, China) was administered intravenously into the tail vein of rats in the CIN and NAC+CIN groups every $15 \mathrm{~min}$, as described by Yokomaku et al (13). Rats in the NAC+CIN group received intraperitoneal injection of $10 \mathrm{mg} / \mathrm{kg}$ NAC (Sigma-Aldrich; Merck Millipore) $12 \mathrm{~h}$ prior to INDO treatment. Rats in the CON group received the same amount of saline injections into their abdomen and tail vein. Rats in the NAC group were pretreated with $10 \mathrm{mg} / \mathrm{kg}$ NAC by intraperitoneal injection, and the same amount of saline as delivered to the CIN group was administered $12 \mathrm{~h}$ later.

Measurement of Scr and serum NGAL. Rats were sacrificed at 2, 12, 24, 48 and $72 \mathrm{~h}$ by anesthetic overdose (10\% chloral hydrate, $0.5 \mathrm{ml} / 100 \mathrm{~g}$; Sangon Biotech Co., Ltd., Shanghai, China) after the procedure. Blood samples were harvested and centrifuged at $1,500 \times \mathrm{g}$ for $15 \mathrm{~min}$ at $4^{\circ} \mathrm{C}$. Scr values was measured by an automatic biochemical analyzer (Olympus Corp., Tokyo, Japan), and the concentration of serum NGAL was evaluated via an enzyme linked immunosorbent assay (ELISA) with an ELISA kit (Westang Biotech, Shanghai, China).

Hemotoxylin and eosin (HE) staining and tubular injury score. Kidneys were harvested and separated into two pieces, one of which was fixed in $10 \%$ formalin for $24 \mathrm{~h}$, and the other was kept at $-80^{\circ} \mathrm{C}$ for subsequent western blot analysis and to study oxidative status. The fixed piece of kidney was processed and embedded in paraffin, cut into $4-\mu \mathrm{m}$-thick section, embedded in paraffin sections and stained with HE. Biopsies were examined by light microscopy in a blinded manner, and scored with a semi-quantitative scale designed to evaluate the degree of tubular injury, according to the tubular necrosis, tubular dilatation and/or atrophy, inflammatory cell infiltration, or cellular edema. Injury was graded on a scale: 0, normal kidney; 1, minimal damage (unicellular, patchy isolated damage); 2 , mild damage $(<25 \%)$; 3 , moderate damage (25-50\%); and 4, severe damage ( $>50 \%)$ (14). A minimum of 10 fields at $\mathrm{x} 400$ magnification were assessed and graded in each biopsy.

Immunohistochemistry of NGAL. Following deparaffinization, antigen retrieval was performed in citrate buffer. Non-specific binding was blocked with $0.3 \% \mathrm{H}_{2} \mathrm{O}_{2}$ for $10 \mathrm{~min}$ and, after blocking for 30 min with $10 \%$ goat serum at room temperature, anti-NGAL antibody (1:150 in PBS; PB0641; Boster Biological Technology, Ltd., Wuhan, China) was applied overnight at $4^{\circ} \mathrm{C}$. Samples were washed in buffer and incubated with the goat anti-rabbit IgG-Biotin secondary antibody (1:200 in PBS; BA1003; Boster Biological Technology, Ltd.) for $30 \mathrm{~min}$, followed by the diaminobenzidine-peroxidase reaction and counterstaining with hematoxylin. Negative control sections were stained under identical conditions by omitting the primary antibody instead of PBS. Stained sections were subsequently visualized using light microscopy at x 200 magnification and the integrated optical density (IOD) was measured using Image-Pro Plus 6.0 (Media Cybernetics, Inc., Rockville, MD, USA).

Western blotting. Total protein extraction was performed by homogenization and centrifugation at 1,500 x $\mathrm{g}$ for $20 \mathrm{~min}$ at $4^{\circ} \mathrm{C}$, and the concentration of the protein was determined by the bicinchoninic acid assay (BCA) kit (Biyuntian Biotechnological Co., Shanghai, China). Protein samples were separated by $12 \%$ SDS-PAGE and transferred onto nitrocellulose membranes (Bio-Rad Laboratories, Inc., Hercules, CA, USA), and blocked with 5\% skimmed milk for 2 h. Primary antibodies against rabbit polyclonal anti-NGAL (1:1,000; ab63929; Abcam, Cambridge, UK) and sheep anti-mouse anti-GAPDH (1:500; sc-25778; Santa Cruz Biotechnology, Inc., Dallas, TX, USA) were respectively added, and incubated at $4^{\circ} \mathrm{C}$ overnight. Following washing in TBST for 5 min three times, the membranes were then incubated with secondary antibody (1:5,000; P/N926-32231; ODYSSEY; LI-COR Biosciences, Inc., Lincoln, NE, USA) for $2 \mathrm{~h}$ in the dark. Protein bands were analyzed via an ODYSSEY Laser Imaging system (LI-COR Biosciences, Inc.).

Renal oxidative stress. Renal cortex was homogenized with ice-cold saline homogenization buffer, and centrifuged at $1,500 \mathrm{x} g$ for $20 \mathrm{~min}$ at $4^{\circ} \mathrm{C}$. The supernatant was analyzed by commercial malondialdehyde (MDA) and superoxide dismutase (SOD) kits (cat. nos. A003-1 and A001-3, respectively; Jiancheng Bioengineering Institute, China). MDA levels were measured via the thiobarbituric acid reactive substances method (15). SOD activity was measured by a method based on the nitro blue tetrazolium (NBT) reduction rate. One unit for SOD activity was expressed as the enzyme protein amount that was able to cause a $50 \%$ inhibition in NBT reduction rate (15). Sample absorbances were measured at $532 \mathrm{~nm}$ and $550 \mathrm{~nm}$ respectively. Protein levels of the supernatants were measured using a BCA assay kit.

Statistical analysis. Statistical analysis was performed using SPSS 17.0 software (SPSS Inc., Chicago, IL, USA). All data were expressed as mean \pm standard deviation. Statistical comparisons were executed by one-way analysis of variance followed by Student-Newman-Keuls analysis. $\mathrm{P}<0.05$ was considered to indicate a statistically significant difference.

\section{Results}

Serum Scr and NGAL values. At 2, 12 and $24 \mathrm{~h}$ following administration, there were no significant differences in the Scr values among the four groups $(\mathrm{P}>0.05)$. Scr levels significantly increased in the CIN group at 48 and $72 \mathrm{~h}(\mathrm{P}<0.05)$, which was significantly higher than the NAC+CIN group at the same time point $(\mathrm{P}<0.05$; Fig. 1). However, compared 
Table I. Renal injury score of each group $(n=30)$ at the indicated time points.

\begin{tabular}{lcccl}
\hline Groups & CON & CIN & NAC & NAC $+C I N$ \\
\hline $2 \mathrm{~h}$ & $0.19 \pm 0.03$ & $0.28 \pm 0.08$ & $0.21 \pm 0.07$ & $0.21 \pm 0.07$ \\
$12 \mathrm{~h}$ & $0.21 \pm 0.04$ & $1.94 \pm 0.21^{\mathrm{a}}$ & $0.17 \pm 0.06^{\mathrm{b}}$ & $1.40 \pm 0.16^{\mathrm{a}, \mathrm{b}}$ \\
$24 \mathrm{~h}$ & $0.18 \pm 0.07$ & $2.42 \pm 0.66^{\mathrm{a}}$ & $0.21 \pm 0.08^{\mathrm{b}}$ & $1.43 \pm 0.08^{\mathrm{a}, \mathrm{b}}$ \\
$48 \mathrm{~h}$ & $0.26 \pm 0.06$ & $2.60 \pm 0.65^{\mathrm{a}}$ & $0.20 \pm 0.02^{\mathrm{b}}$ & $1.79 \pm 0.24^{\mathrm{a}, \mathrm{b}}$ \\
$72 \mathrm{~h}$ & $0.26 \pm 0.04$ & $2.02 \pm 0.16^{\mathrm{a}}$ & $0.25 \pm 0.06^{\mathrm{b}}$ & $1.25 \pm 0.15^{\mathrm{a}, \mathrm{b}}$ \\
\hline
\end{tabular}

${ }^{\text {ap }}<0.05$ vs. the CON group at the same time point; ${ }^{\text {b }}<0.05$ vs. the $\mathrm{CIN}$ at the same time point. Data are presented as the mean \pm standard deviation. CON, control; CIN, contrast-induced nephropathy; $\mathrm{NAC}, \mathrm{N}$-acetylcysteine.

Table II. Integrated optical density value of each group $(\mathrm{n}=30)$ at the indicated time points.

\begin{tabular}{lcccc}
\hline Groups & CON & CIN & NAC & NAC+CIN \\
\hline $2 \mathrm{~h}$ & $2.81 \pm 0.27$ & $15.54 \pm 3.31^{\mathrm{a}}$ & $2.67 \pm 0.19^{\mathrm{b}}$ & $10.90 \pm 1.42^{\mathrm{a}, \mathrm{b}}$ \\
$12 \mathrm{~h}$ & $2.34 \pm 0.17$ & $32.30 \pm 3.31^{\mathrm{a}}$ & $2.43 \pm 0.25^{\mathrm{b}}$ & $20.39 \pm 2.2^{\mathrm{a}, \mathrm{b}}$ \\
$24 \mathrm{~h}$ & $2.79 \pm 0.32$ & $55.74 \pm 3.62^{\mathrm{a}}$ & $2.58 \pm 0.34^{\mathrm{b}}$ & $32.94 \pm 0.31^{\mathrm{a}, \mathrm{b}}$ \\
$48 \mathrm{~h}$ & $2.42 \pm 0.39$ & $27.38 \pm 2.15^{\mathrm{a}}$ & $2.32 \pm 0.14^{\mathrm{b}}$ & $16.55 \pm 2.46^{\mathrm{a}, \mathrm{b}}$ \\
$72 \mathrm{~h}$ & $2.47 \pm 0.29$ & $13.41 \pm 0.10^{\mathrm{a}}$ & $2.42 \pm 0.20^{\mathrm{b}}$ & $8.57 \pm 0.55^{\mathrm{a}, \mathrm{b}}$ \\
\hline
\end{tabular}

${ }^{\mathrm{a}} \mathrm{P}<0.05$ vs. the $\mathrm{CON}$ group at the same time point, ${ }^{\mathrm{b}} \mathrm{P}<0.05$ vs. the $\mathrm{CIN}$ at the same time point. Data are presented as the mean \pm standard deviation. CON, control; CIN, contrast-induced nephropathy; $\mathrm{NAC}, \mathrm{N}$-acetylcysteine.

with the CON group, the value of serum NGAL in the CIN group had markedly increased, with a significant increase at $2 \mathrm{~h}$ after administration ( $\mathrm{P}<0.05$; Fig. 2). NGAL values in the $\mathrm{NAC}+\mathrm{CIN}$ group were significantly decreased, as compared with the CIN group $(\mathrm{P}<0.05$; Fig. 2).

HE staining and renal injury score. In contrast-injected rats, vacuolar degeneration of tubular epithelial cells, tubular dilation, protein cast (protein deposition in the tubular lumen), tubular brush border loss, increased epithelial cell shedding, and necrosis of partial tubular epithelial cells were observed (Fig. 3). Semi-quantitative analysis demonstrated no difference between the renal injury scores of the NAC and CON groups. Renal injury scores in the NAC+CIN group were higher than that of the CON group, but significantly lower than that of the CIN group $(\mathrm{P}<0.05$; Table I).

Immunohistochemistry. Immunohistochemistry findings demonstrated that NGAL was predominantly expressed in the cytoplasm of proximal tubular cells (Fig. 4). Compared with the CON group, the positive staining IOD value of NGAL significantly increased $2 \mathrm{~h}$ after administration in the CIN group $(\mathrm{P}<0.05$; Table II), and was significantly higher than that of the NAC+CIN group at same time point $(\mathrm{P}<0.05$; Table II).

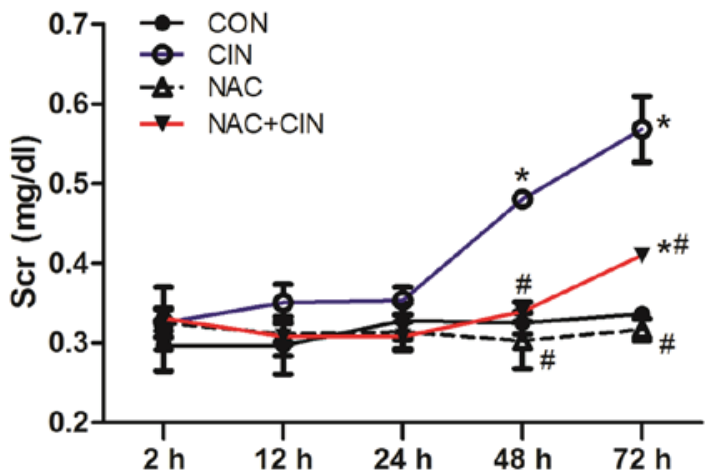

Figure 1. Scr values in each group. ${ }^{*} \mathrm{P}<0.05$ vs. the CON group at the same time point; ${ }^{\#} \mathrm{P}<0.05$ vs. the $\mathrm{CIN}$ at same time point. Data are presented as the mean \pm standard deviation. CON, control; CIN, contrast-induced nephropathy; NAC, N-acetylcysteine.

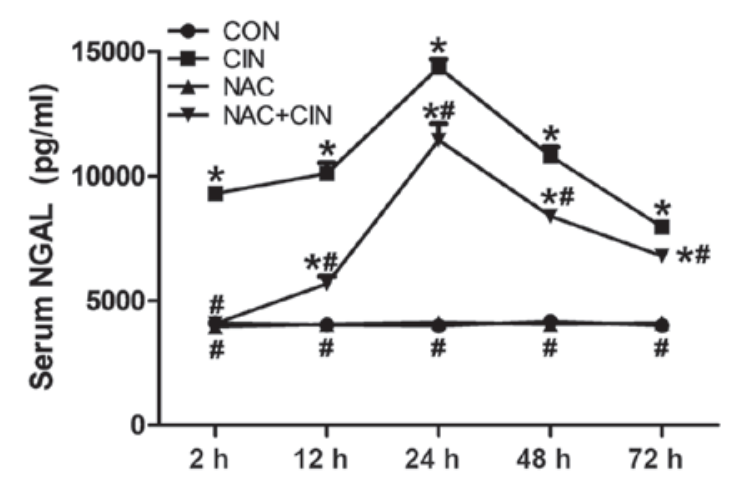

Figure 2. Serum NGAL levels in each group. Data are presented as the mean \pm standard deviation. ${ }^{*} \mathrm{P}<0.05$ vs. the CON group at the same time point; ${ }^{\#} \mathrm{P}<0.05$ vs. the CIN group at same time point. CON, control; CIN, contrast-induced nephropathy; NAC, N-acetylcysteine.

Western blotting. According to the western blotting results, the expression levels of NGAL in the kidney tissue of the CIN group began to significantly increase $2 \mathrm{~h}$ after the procedure $(\mathrm{P}<0.05)$, and were significantly decreased in the NAC+CIN group at the same time point $(\mathrm{P}<0.05)$. No significant differences were detected between the NAC and CIN groups ( $\mathrm{P}>0.05$; Fig. 5).

Oxidative stress indicators. As shown in Tables III and IV, a significant decline of kidney SOD was observed in CIN rats compared with the CON group $(\mathrm{P}<0.05)$, and there was also a significant decline between the CIN and NAC+CIN groups $(\mathrm{P}<0.05)$. This was accompanied by an increase of MDA in the CIN group, which was significantly higher than the other three groups $(\mathrm{P}<0.05)$.

\section{Discussion}

CIN has become the third leading cause of hospital-acquired acute renal failure, accounting for 10-25\% of all acquired acute renal damage (16). CIN is defined as an increase in serum creatinine by $0.3 \mathrm{mg} / \mathrm{dl}$ within $48 \mathrm{~h}$ (16). However, serum creatinine is an unreliable indicator of acute changes in renal function (17). Therefore, a biomarker that can accurately reflect the kidney injury of early stage is urgently required, to improve the prognosis of patients. 
A

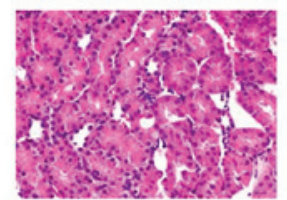

B

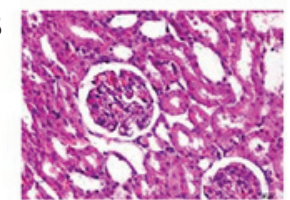

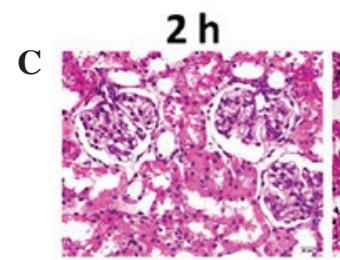

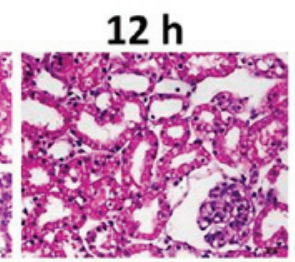

D
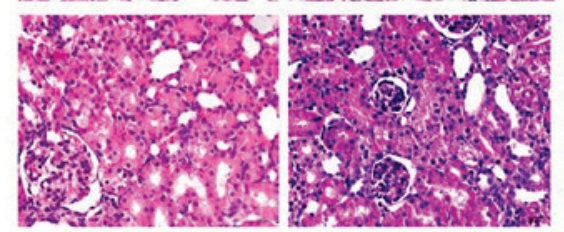

$24 \mathrm{~h}$
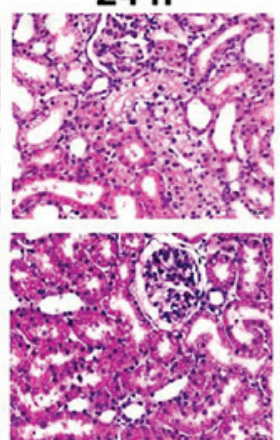

$48 \mathrm{~h}$
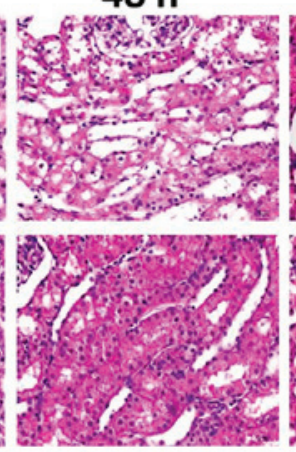

$72 \mathrm{~h}$
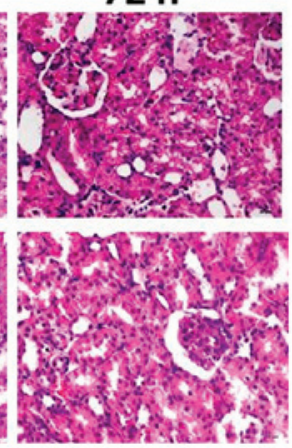

Figure 3. Hemotoxylin and eosin staining of each group at the indicated time points (magnification, $\mathrm{x} 400)$. (A) Control, (B) N-acetylcysteine, (C) contrast-induced nephropathy, and (D) N-acetylcysteine + contrast-induced nephropathy groups. As the control and N-acetylcysteine groups demonstrated no significant differences in staining at any time point, one time point was randomly chosen in each group.
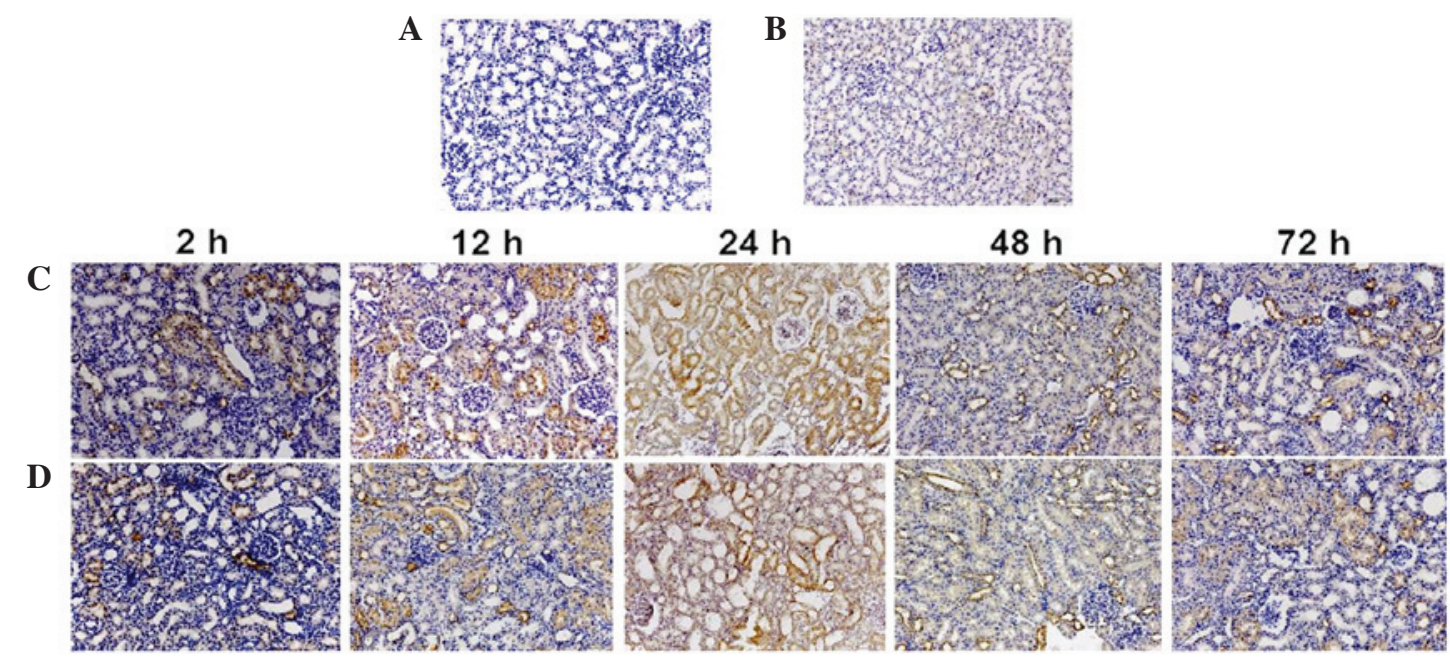

Figure 4. Immunohistochemistry of each group at the indicated time points (magnification, x200). (A) Control, (B) N-acetylcysteine, (C) contrast-induced nephropathy and (D) N-acetylcysteine + contrast-induced nephropathy groups. As the control and N-acetylcysteine groups demonstrated no significant differences in immunohistochemistry at any time point, one time point was randomly chosen in each group.

NGAL is a protein with a small molecular weight $(25 \mathrm{kDa})$, which is a member of lipocalin family, that can be covalently combined with neutrophil gelatinase (18). NGAL is present in various organs; under normal conditions, NGAL remains at a low expression level in the kidneys, trachea and epithelial cells of the gastrointestinal tract. However, when ischemia or ischemic reperfusion injury occurs, the secretion of NGAL in the thick ascending limb segment of renal tubular increases rapidly. In the present study, a rat model of CIN was established and treated with NAC. The expression of NGAL was subsequently detected in the kidney and serum and the presence of kidney damage was assessed, in order to explore whether NGAL may be used as an early diagnostic marker of CIN, and whether NAC has a protective effect on the kidneys. The present data showed that significant kidney damage occurred in the rat model $12 \mathrm{~h}$ after the injection of contrast medium; however, Scr values did not increase until $48 \mathrm{~h}$ after injection. Meanwhile, immunohistochemistry and western blot analysis indicated that NGAL expression levels in the kidney had increased by $2 \mathrm{~h}$ post-injection, and peaked at $24 \mathrm{~h}$. ELISA analysis demonstrated that the serum level of NGAL increased $2 \mathrm{~h}$ after the injection, demonstrating the same variation trends as NGAL in the kidney. When Mishra et al (19) conducted a study of 71 children undergoing cardiac surgery, the NGAL level in serum and urine significantly increased $2 \mathrm{~h}$ after acute kidney injury; however, but Scr levels did not change until $24 \mathrm{~h}$ later. Bennett et al (20) drew the same conclusion, and the present findings were consistent. Therefore, NGAL is a superior indicator for the kidney injury of early stage than Scr.

Due to the high incidence and mortality rates of CIN, it is important that a strategy is adopted to prevent the occurrence of $\mathrm{CIN}$ in high-risk patients. Hydration therapy has been demonstrated to be effective $(21,22)$; however, individuals who do not have good cardiac function, cannot receive a large liquid infusion, thus hydration therapy is not viable. A previous study has demonstrated the harm of hydration therapy (23). Studies have 
Table III. The level of SOD in renal tissue (U/mgprot).

\begin{tabular}{lrrrr}
\hline Groups & CON $(n=30)$ & CIN $(n=30)$ & NAC $(n=30)$ & NAC+CIN $(n=30)$ \\
\hline $2 \mathrm{~h}$ & $144.39 \pm 1.786$ & $113.46 \pm 2.114^{\mathrm{a}}$ & $150.32 \pm 4.968^{\mathrm{b}}$ & $130.03 \pm 0.791^{\mathrm{a}, \mathrm{b}}$ \\
$12 \mathrm{~h}$ & $160.64 \pm 0.807$ & $97.32 \pm 0.833^{\mathrm{a}}$ & $167.15 \pm 1.576^{\mathrm{b}}$ & $105.08 \pm 0.356^{\mathrm{a}, \mathrm{b}}$ \\
$24 \mathrm{~h}$ & $124.88 \pm 4.521$ & $67.20 \pm 0.464^{\mathrm{a}}$ & $128.78 \pm 2.474^{\mathrm{b}}$ & $97.32 \pm 0.833^{\mathrm{a}, \mathrm{b}}$ \\
$48 \mathrm{~h}$ & $159.04 \pm 1.187$ & $90.78 \pm 0.672^{\mathrm{a}}$ & $163.63 \pm 1.018^{\mathrm{b}}$ & $100.36 \pm 0.858^{\mathrm{a}, \mathrm{b}}$ \\
$72 \mathrm{~h}$ & $137.78 \pm 1.383$ & $107.15 \pm 1.568^{\mathrm{a}}$ & $139.45 \pm 1.929^{\mathrm{b}}$ & $125.00 \pm 1.609^{\mathrm{a}, \mathrm{b}}$ \\
\hline
\end{tabular}

Data are presented as the mean \pm standard deviation. ${ }^{\mathrm{a}} \mathrm{P}<0.05$ vs.the $\mathrm{CON}$ group at the same time point; ${ }^{\mathrm{b}} \mathrm{P}<0.05$ vs. the $\mathrm{CIN}$ group at the same time point. SOD, superoxide dismutase; CON, control; CIN, contrast-induced nephropathy; NAC, N-acetylcysteine.

Table IV. The level of MDA in renal tissue (nmol/mgprot).

\begin{tabular}{lcccc}
\hline Groups & CON $(\mathrm{n}=30)$ & $\mathrm{CIN}(\mathrm{n}=30)$ & $\mathrm{NAC}(\mathrm{n}=30)$ & $\mathrm{NAC}+\mathrm{CIN}(\mathrm{n}=30)$ \\
\hline $2 \mathrm{~h}$ & $2.42 \pm 0.101$ & $3.01 \pm 0.133^{\mathrm{a}}$ & $2.33 \pm 0.188^{\mathrm{b}}$ & $2.52 \pm 0.215^{\mathrm{b}}$ \\
$12 \mathrm{~h}$ & $2.76 \pm 0.215$ & $3.19 \pm 0.175^{\mathrm{a}}$ & $2.72 \pm 0.136^{\mathrm{b}}$ & $3.25 \pm 0.303^{\mathrm{a}, \mathrm{b}}$ \\
$24 \mathrm{~h}$ & $2.26 \pm 0.122$ & $5.17 \pm 0.257^{\mathrm{a}}$ & $2.13 \pm 0.131^{\mathrm{b}}$ & $4.16 \pm 0.258^{\mathrm{a}, \mathrm{b}}$ \\
$48 \mathrm{~h}$ & $3.00 \pm 0.293$ & $4.51 \pm 0.309^{\mathrm{a}}$ & $2.86 \pm 0.252^{\mathrm{b}}$ & $3.96 \pm 0.199^{\mathrm{a}, \mathrm{b}}$ \\
$72 \mathrm{~h}$ & $2.36 \pm 0.152$ & $3.33 \pm 0.154^{\mathrm{a}}$ & $2.22 \pm 0.028^{\mathrm{b}}$ & $3.14 \pm 0.088^{\mathrm{a}, \mathrm{b}}$ \\
\hline
\end{tabular}

Data are presented as the mean \pm standard deviation. ${ }^{a} \mathrm{P}<0.05$ vs. the $\mathrm{CON}$ group at the same time point; ${ }^{\mathrm{b}} \mathrm{P}<0.05$ vs. the $\mathrm{CIN}$ group at the same time point. MDA, malondialdehyde; CON, control; CIN, contrast-induced nephropathy; NAC, N-acetylcysteine.

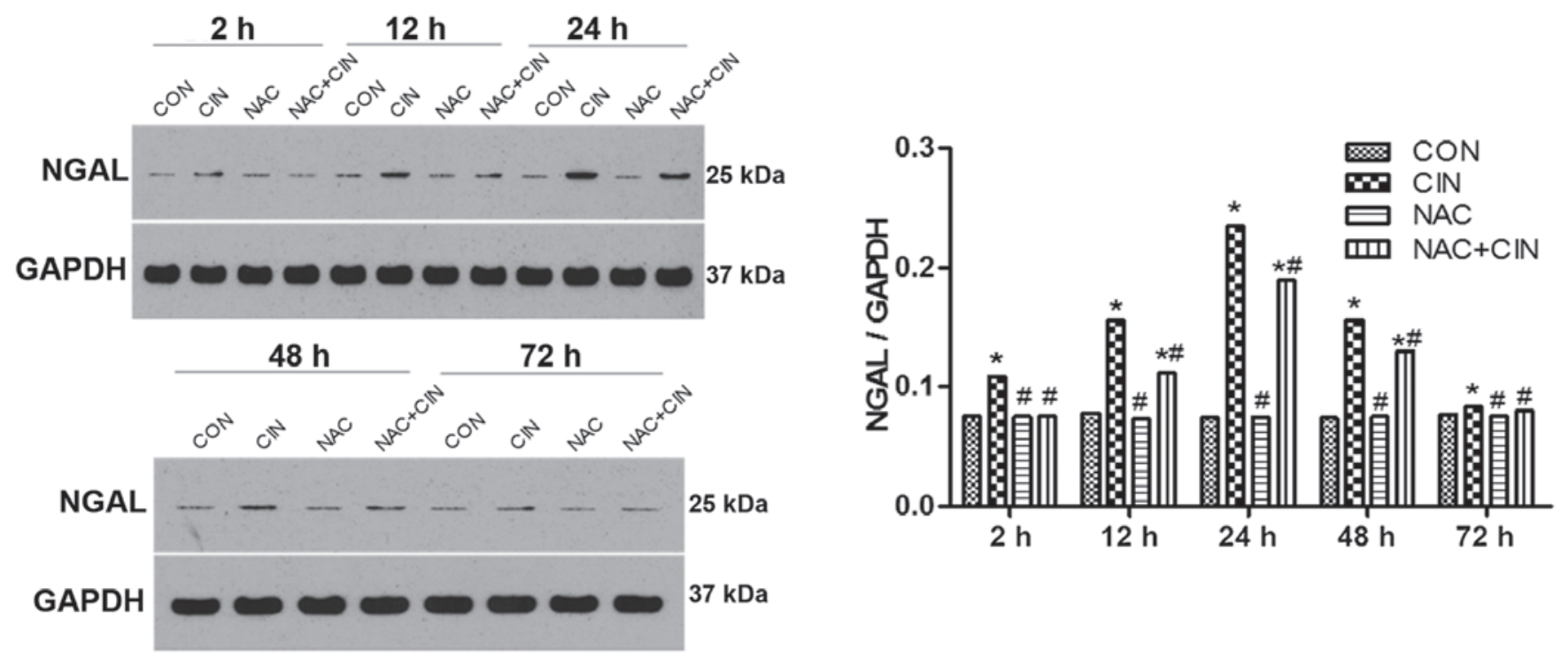

Figure 5. Western blot of each group at the indicated time points. Data are presented as the mean \pm standard deviation. ${ }^{*} \mathrm{P}<0.05$ vs. the $\mathrm{CON}$ group at the same time point; ${ }^{\text {P }}<0.05$ vs. the $\mathrm{CIN}$ at the same time point. CON, control; CIN, contrast-induced nephropathy; NAC, N-acetylcysteine.

shown that oxidative stress has an important role in CIN (24-26). In oxidative stress, radicals reacts with lipids to form MDA. MDA is cytotoxic, and can cause the polymerization of protein and nucleic acid. There are two types of antioxidant systems in the human body, antioxidant enzyme systems and non-enzymatic antioxidant system. SOD is an important member of the antioxidant enzyme family. These enzymes constitute one of the primary defense mechanisms of cells against oxidative stress and serve a role in the pathogenesis of certain invasive bacteria.
NAC has been used as a type of mucolytics to treat respiratory diseases in clinical settings for $\geq 30$ years. It is a precursor of intracellular reduced glutathione, with various biological activities, including interfering radical generation, clearing radicals, regulating intracellular metabolic activities and preventing DNA damage $(8,27)$. In the present study, NAC was investigated for its potential to reduce the kidney damage caused by contrast agents. Scr values in the CIN group has significantly increased $48 \mathrm{~h}$ after the injection of contrast 
medium; however, at the same time point, the difference between the NAC+CIN and CON groups was not statistically significant. Scr values in the NAC+CIN group did not significantly increase until $72 \mathrm{~h}$ later, and remained lower than that of the CIN group at the same time point. Conversely, HE staining of the kidney tissue demonstrated that the NAC+CIN group exhibited markedly reduced pathological manifestations of renal injury compared with the CIN group, such as brush border disappearance, vacuolization, tubular necrosis and protein deposition. Meanwhile, the renal injury scores of the NAC+CIN group were significantly lower than those of the CIN group at each time point. These findings suggest that NAC treatment may be a useful method to protect the kidneys from the injury caused by contrast agents.

According to the present data, a decline in kidney SOD levels was observed in the CIN group compared with the $\mathrm{CON}$ or NAC+CIN groups, accompanied by an increase of kidney MDA, which was significantly higher than the other three groups. This indicates that NAC altered the influence of contrast agent on renal oxidative stress. No significant differences were detected between all the indicators of the NAC and CON groups. Therefore, these findings indicated that NAC did not have a significant impact on normal kidneys, but may inhibit the contrast agent-induced oxidative stress damage in the kidney.

In conclusion, the present study demonstrated that NGAL may be a superior indicator of early stage kidney injury than the conventional diagnostic indicator, Scr. Prevention of NAC was able to reduce the kidney damage caused by contrast agents, and this may be associated with the inhibition of oxidative stress. The present research demonstrated the feasibility of a novel indicator for the prevention of kidney injury, and provides a new choice for the prevention of contrast-induced nephropathy.

\section{References}

1. Nash K, Hafeez A and Hou S: Hospital-acquired renal insuffciency. Am J Kidney Dis 39: 930-936, 2002.

2. Bonventre JV, Vaidya VS, Schmouder R, Feig P and Dieterle F: Next-generation biomarkers for detecting kidney toxicity. Nature Biotechnol 28: 436-440, 2010.

3. Patsaoura A, Tatsi E, Margeli A, Kanavaki I, Delaporta P, Kyriakopoulou D, Kouraklis-Symeonidis A, Kattamis A, Papassotiriou I: Plasma neutrophil gelatinase-associated lipocalin levels are markedly increased in patients with non-transfusion-dependent thalassemia: Lack of association with markers of erythropoiesis, iron metabolism and renal function.Clin Biochem 47: 1060-1064, 2014.

4. Finn WF: The clinical and renal consequences of contrast-induced nephropathy. Nephrol Dial Transplant 21: i2-i10, 2006.

5. Tepel M, Aspelin P and Lameire N: Contrast-induced nephropathy: A clinical and evidence-based approach. Circulation 113: 1799-1806, 2006.

6. Mehran R and Nikolsky E: Contrast-induced nephropathy: Definition, epidemiology, and patients at risk. Kidney Int Suppl: S11-S15, 2006

7. Goldenberg I and Matetzky S: Nephropathy induced by contrast media: Pathogenesis, risk factors and preventive strategies. CMAJ 172: 1461-1471, 2005.

8. Briguori C, Tavano D and Colombo A: Contrast agent-associated nephrotoxicity. Prog Cardiovasc Dis 45: 493-503, 2003.
9. Toprak $\mathrm{O}$ and Cirit M: Risk factors for contrast-induced nephropathy. Kidney Blood Press Res 29: 84-93, 2006.

10. Katzberg RW: Urography into the 21st century: New contrast media, renal handling, imaging characteristics, and nephrotoxicity. Radiology 204: 297-312, 1997.

11. Tumlin J, Stacul F, Adam A, Becker CR, Davidson C, Lameire $\mathrm{N}$ and McCullough PA; CIN Consensus Working Panel: Pathophysiology of contrast-induced nephropathy. Am J Cardiol 98: 14K-20K, 2006.

12. Perrin T, Descombes E and Cook S: Contrast-induced nephropathy in invasive cardiology. Swiss Med Wkly 142: w13608, 2012.

13. Yokomaku Y, Sugimoto T, Kume S, Araki S, Isshiki K, Chin-Kanasaki M, Sakaguchi M, Nitta N, Haneda M, Koya D, et al: Asialoerythropoietin prevents contrast-induced nephropathy. J Am Soc Nephrol 19: 321-328, 2008.

14. James MT, Ghali WA, Tonelli M, Faris P, Knudtson ML, Pannu N, Klarenbach SW, Manns BJ and Hemmelgarn BR: Acute kidney injury following coronary angiography is associated with a long-term decline in kidney function. Kidney Int 78: 803-809, 2010.

15. James MT, Ghali WA, Knudtson ML, Ravani P, Tonelli M, Faris P, Pannu N, Manns BJ, Klarenbach SW, Hemmelgarn BR, et al: Associations between acute kidney injury and cardiovascular and renal outcomes after coronary angiography. Circulation 123: 409-416, 2011.

16. Kidney Disease: Improving Global Outcomes (KDIGO) Acute Kidney Injury Work Group. KDIGO clinical practice guideline for acute kidney injury. Kidney Int Suppl 2: 1-138, 2012.

17. Bellomo R, Kellum JA and Ronco C: Defining acute renal failure: Physiological principles. Intensive Care Med 30: 33-37, 2004.

18. Cowland JB and Borregaard N: Molecular characterization and pattern of tissue expression of the gene for NGAL from humans. Genomics 45: 17-23, 1997.

19. Mishra J, Mor IK, Ma Q, Kelly C, Yang J, Mitsnefes M, Barasch J and Devarajan P: Amelioration of ischemic acute renal injury by neutrophil gelatinase-associated lipocalin. J Am Soc Nephrol 15: 3073-3082, 2004.

20. Bennett M, Dent CL, Ma Q, Dastrala S, Grenier F, Workman R, Syed H, Ali S, Barasch J and Devarajan P: Urine NGAL predicts severity of acute kidney injury after cardiac surgery: A prospective study. Clin J Am Soc Nephrol 3: 665-673, 2008.

21. Navaneethan SD, Singh S, Appasamy S, Wing RE and Sehgal AR: Sodium bicarbonate therapy for prevention of contrast-induced nephropathy: A systematic review and meta-analysis. Am J Kidney Dis 53: 617-627, 2009.

22. Recio-Mayoral A, Chaparro M, Prado B, Cózar R, Méndez I, Banerjee D, Kaski JC, Cubero J and Cruz JM: The reno-protective effect of hydration with sodium bicarbonate plus $\mathrm{N}$-acetylcysteine in patients undergoing emergency percutaneous coronary intervention: The RENO study. J Am Coll Cardiol 49: 1283-1288, 2007.

23. Reinecke H, Fobker M, Wellmann J, Becke B, Fleiter J, Heitmeyer C, Breithardt G, Hense HW and Schaefer RM: A randomized controlled trial comparing hydration therapy to additional hemodialysis or $\mathrm{N}$-acetylcysteine for the prevention of contrast medium-induced nephropathy: The Dialysis-versus-Diuresis (DVD) Trial. Clin Res Cardiol 96: 130-139, 2007.

24. Toprak O, Cirit M, Tanrisev M, Yazici C, Canoz O, Sipahioglu M, Uzum A, Ersoy R and Sozmen EY: Preventive effect of nebivolol on contrast-induced nephropathy in rats. Nephrol Dial Transplant 23: 853-859, 2008.

25. Goodman A, Olszanecki R, Yang LM, Quan S, Li M, Omura S, Stec DE and Abraham NG: Heme oxygenase-1 protects against radiocontrast-induced acute kidney injury by regulating anti-apoptotic proteins. Kidney Int 72: 945-953, 2007.

26. Katholi RE, Woods WT Jr, Taylor GJ, Deitrick CL, Womack KA, Katholi CR and McCann WP: Oxygen free radicals and contrast nephropathy. Am J Kidney Dis 32: 64-671, 1998.

27. Heyman SN, Rosen S, Khamaisi M, Idée JM and Rosenberger C: Reactive oxygen species and the pathogenesis of radiocontrast-induced nephropathy. Invest Radiol 45: 188-195, 2010 . 